International Journal of Pure and Applied Mathematics

Volume 85 No. 2 2013, 255-263

ISSN: 1311-8080 (printed version); ISSN: 1314-3395 (on-line version)

url: http://www.ijpam.eu

doi: http://dx.doi.org/10.12732/ijpam.v85i2.6

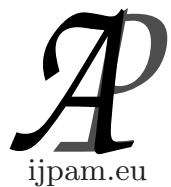

\title{
TOWARDS URYSOHN'S LEMMA IN MINIMAL STRUCTURES
}

\author{
M. Singha ${ }^{1}$, S. De Sarkar ${ }^{2}$ \\ Department of Mathematics \\ University of North Bengal \\ North Bengal, INDIA
}

\begin{abstract}
With necessary modifications and corrections of some results in the existing literature on minimal structures we propose in this paper a development in the related theory that enables us, along with other important results, to establish a version of Urysohn's lemma in this setting. In doing so various separation axioms and different kinds of continuity in the replacement of topology, generalized topology or weak structure by minimal structure which are closely related to Urysohn's lemma have been studied.
\end{abstract}

AMS Subject Classification: 54A05, 54A10, 54A20, 54C08, 54D10, 54D30 Key Words: minimal structure, $m$-open, $m$-closed, $i_{m}, c_{m}, i^{*}{ }_{m}, c^{*}{ }_{m}, m-T_{1}$, $m-T_{2}, m$-regular MSS, $m$-normal MSS, $M$-continuity, $M^{*}$-continuity, lowersemi-continuity, upper-semi-continuity

\section{Introduction and Preliminaries}

Minimal structure, as the name suggests, involves least possible requirements for a class of subsets of a nonempty set to have some "structure" from the view point of topological studies. This unique concept, emerged in 2000 (V. Popa and T. Noiri [7]), drew the attention of a number of researchers in the related field. Very recent, it was further studied by Carlos Carpintero, Ennis Rosas, Margot Salas [3], A. Pushpalatha, E. Subha [4], R. Parimelazhagan, K. Balachandran,

Received: November 13, 2012

(C) 2013 Academic Publications, Ltd.

$\S$ Correspondence author url: www.acadpubl.eu 
N. Nagaveni [5], Sunisa Buadong, Chokchai Viriyapong, Chawalit Boonpok [6], Won Keun Min, Young Key Kim [7] etc.. We extend the field by introducing notions of various separation axioms, continuity and established the Urysohn's lemma in this context. At first we give some basic definitions and outline of the results relavent to this extension. As in $[2,3,4,5,6,7]$ a family $m$ of subsets of a non-empty set $X$ containing the empty subset and the whole set is called a minimal structure and we call the ordered pair $(X, m)$ a minimal structured space (briefly MSS). Elements of a minimal structure $m$ on $X$ are called $m$-open sets and their complements are called $m$-closed sets. For any $A \subset X, i_{m}(A)(m-$ Int in [7]) denotes the union of all $m$-open sets contained in $A$ and $c_{m}(A)(m-C l$ in [7]) denotes the intersection of all $m$-closed sets containing $A$; presence of the empty set in $m$ well-defines $i_{m}(A)$ and $c_{m}(A) . i_{m}$ is contractive $c_{m}$ is expansive, both are monotonic and idempotent and their relationship is $i_{m}(A)=X-c_{m}(X-A)$, for any $A \subset X . x \in i_{m}(A)$ iff there is an $m$-open set $B \subset A$ containing $x$ and $x \in c_{m}(A)$ iff $B \cap A \neq \phi$ whenever $x \in B \in m$. All these are similar results to other topology-like structures (topology or generalized topology or weak structure) but unlike in topology or generalized topology $i_{m}(A)$ may not be $m$-open and $c_{m}(B)$ may not be $m$-closed, for $A, B \subset X$; though they will be so if $A$ is $m$-open and $B$ is $m$-closed. In the present work we have shown that how this dissimilarities propagate differences in separation axioms, continuity and Urysohn's lemma.

\section{Correction of Some Results Appeared in [7]}

Let's begin with the following example;

Example 2.1. Let us consider the minimal structure $m_{X}=\{\phi,\{a, b\},\{b, c\}$, $X\}$ on $X=\{a, b, c\}, A=\{a, b\}, B=\{b, c\}, C=\{c\}$ and $D=\{a\}$ then $m-\operatorname{Int}(A)=A, m-\operatorname{Int}(B)=B, m-\operatorname{Int}(A \cap B)=\phi, m-C l(C)=C$, $m-C l(D)=D$ and $m-C l(C \cup D)=X$.

This shows that the statement in Theorem 2.5 (5) [7] is not true and it must be replaced by $m-\operatorname{Int}(A \cap B) \subset m-\operatorname{Int}(A) \cap m-\operatorname{Int}(B)$ and $m-C l(A) \cup$ $m-C l(B) \subset m-C l(A \cup B)$.

In the proof of the Theorem 3.8 [7] and Theorem 4.20 [7] monotonicity of the operators $I_{m}$ and $C l_{m}$ have been used but none of them is monotonic as shown in the following example;

Example 2.2. Consider the minimal structure $m_{X}=\{\phi,\{a, b\},\{b, c\},\{c, d\}$, $X\}$ on $X=\{a, b, c, d\}, A=\{a, b, c\}, B=\{a, b\}$ and $C=\{a\}$ then $I_{m}(A)=\phi$, 
$I_{m}(B)=B, C l_{m}(B)=B$ and $C l_{m}(C)=X$.

The statement of the Theorem $3.8[7]$ is wrong because $(3) \Leftrightarrow(4)$ there. The following examples are supporting wittiness.

Example 2.3. Let $X=\{a, b, c\}, Y=\{1,2\}, m_{X}=\{\phi,\{b\}, X\}, m_{Y}=$ $\{\phi,\{2\}, Y\}$ and $f: X \rightarrow Y$ be a function defined by $f(a)=f(c)=1, f(b)=2$; $f$ is $M^{*}$-continuous and Theorem $3.8(3)$ [7] holds but $f\left(C l_{m_{X}}(A)\right)=f(X)=$ $\{1,2\} \nsubseteq\{1\}=C l_{m_{Y}}(\{1\})=C l_{m_{Y}}(f(A))$, where $A=\{c\}$, shows that Theorem 3.8 (4) [7] does not hold.

Example 2.4. Let $X=\{a, b, c\}, Y=\{1,2,3\}, m_{X}=\{\phi,\{b\}, X\}$ and $m_{Y}=\{\phi,\{2\}, Y\}$. Then the function $f: X \rightarrow Y$ defined by $f(a)=f(b)=$ $1, f(c)=2$ is not $M^{*}$-continuous and Theorem 3.8 (3) [7] is not true for this function but it can be verified that Theorem 3.8 (4) [7] holds.

The Theorem 4.20 [7] seems to be incorrect, we put it in the revised form in the following

Theorem 2.1. A bijection $f:\left(X, m_{X}\right) \rightarrow\left(Y, m_{Y}\right)$, where $m_{X}$ and $m_{Y}$ are minimal structures on $X$ and $Y$ respectively, is $M^{*}$-open if and only if $I_{m}\left(f^{-1}(B)\right) \subset f^{-1}\left(I_{m}(B)\right)$ for each $B \subset Y$.

A corrected version of the Theorem 3.8 [7] is included in the Theorem 3.11 of the following Section 3 .

\section{Separation Axioms, Continuity and Urysohn's Lemma}

Definition 3.1. A minimal structured space $(X, m)$ is called $m-T_{1}$ if for any two distinct points $x, y \in X$ there are $m$-open sets $U$ and $V$ so that $x \in U$, $y \in V, x \notin V$ and $y \notin U$.

Example 3.1. Let $X=\{a, b, c\}$. On this $X, m_{1}=\{\Phi, X,\{a\},\{b\},\{c\}\}$, $m_{2}=\{\Phi, X,\{a, b\},\{b, c\},\{c, a\}\}$ are MSs and the corresponding MSSs are $m-$ $T_{1}$.

The above MSSs in Example 3.1 show that a finite MSS may be $m-T_{1}$ without being $P(X)$.

Definition 3.2. Let $(X, m)$ be an MSS. An element $A$ of $P(X)$ is called a fixed point of $c_{m}$ if $c_{m}(A)=A$.

Theorem 3.1. A MSS $(X, m)$ is $m-T_{1}$ iff every singleton is a fixed point of $c_{m}$. 
Proof. If $x \neq y \in c_{m}(\{x\})$ then every $m$-open set containing $y$ will contain $x$ and $(X, m)$ would not be $m-T_{1}$. Thus in a $m-T_{1} \operatorname{MSS}(X, m)$ every singleton is a fixed point of $c_{m}$. Conversely for any two distinct points $x$ and $y$ in a $\operatorname{MSS}(X, m)$, where every singleton is a fixed point of $c_{m}, y \notin c_{m}(\{x\})$ and $x \notin c_{m}(\{y\})$; this implies there are $m$-open sets $U$ and $V$ containing $x$ and $y$ respectively such that $U \bigcap\{y\}=\Phi$ and $V \bigcap\{x\}=\Phi$, so $(X, m)$ is $m-T_{1}$.

Note that those singletons may not be $m$-closed, $\left(X, m_{1}\right)$ in Example 3.1 is one such wetness.

Definition 3.3. An $\operatorname{MSS}(X, m)$ is called $m-T_{2}$ if for any two distinct points $x, y \in X$ there exists disjoint $m$-open sets $U$ and $V$ so that $x \in U, y \in V$.

In Example $3.1\left(X, m_{1}\right)$ is a $m-T_{2}$ but $\left(X, m_{2}\right)$ is not $m-T_{2}$ though it is $m-T_{1}$. Obviously an $m-T_{2}$ MSS is $m-T_{1}$.

Definition 3.4. We say that a sequence $\left\{x_{n}\right\}$ in an $\operatorname{MSS}(X, m)$ converges to $x \in X$ if for any $m$-open set $U$ there exists a positive integer $N$ so that $x \in U$ for all $n \geq N$; in this case $\left\{x_{n}\right\}$ is called a convergent sequence and $x$ is called limit of it.

Theorem 3.2. In an $m-T_{2}$ MSS every convergent sequence has unique limit.

Proof. If $x, y$ are two distinct limits of a sequence $\left\{x_{n}\right\}$ in an $m-T_{2}$ MSS $(X, m)$, then for any two $m$-open sets $U$ and $V$ containing $x$ and $y$ respectively there exists a positive integer $N$ so that $x_{n} \in U \cap V$ for all $n \geq N$ and this contradicts the fact that the MSS is $m-T_{2}$.

Definition 3.5. An $\operatorname{MSS}(X, m)$ is called $m$-regular if for any point $x \in X$ and any $m$-closed set $C$ not containing $x$ there exists disjoint $m$-open sets $U$ and $V$ so that $x \in U$ and $C \subset V$.

Theorem 3.3. If $(X, m)$ is $m$-regular MSS then for any $x \in U \in m$ there exists $V \in m$ so that $x \in V \subset c_{m}(V) \subset U$.

Proof. Let $(X, m)$ be an $m$-regular MSS and $U$ be any $m$-open set, then for any point $x \in U$ the $m$-closed set $X-U$ does not contain $x$, so there exists $m$-open sets $V$ and $E$ such that $x \in V, X-U \subset E$ and $V \cap E=\phi$. This implies $V \subset X-E$ and hence $c_{m}(V) \subset X-E \subset U$, since $X-E$ is $m$-closed. Thus for any $x \in U \in m$ there exists $V \in m$ so that $x \in V \subset c_{m}(V) \subset U$.

But the converse of the Theorem 3.3 is not true as seen in the MSS $\left(X, m_{1}\right)$ of the Example 3.1 which is true in regular topological spaces. 
We call the family $M(x)=\{U ; x \in U \in m\}$, where $m$ is a minimal structure on $X$, a minimal-star at $x$ and by the help of the family $\{M(x) ; x \in X\}$ of minimal-stars we define $i^{*}{ }_{m}(A)=\{x \in A ; A \in M(x)\}\left(=I_{m}(A)\right.$ in [7]) and $c^{*}{ }_{m}(A)=\{x \in X ; X-A \notin M(x)\}\left(=C l_{m}(A)\right.$ in $\left.[7]\right)$. Now $i^{*}{ }_{m}$ agrees with $i_{m}$ in $m$ and $c^{*}{ }_{m}$ agrees with $c_{m}$ in $m^{c}=\{X-U ; U \in m\}$; also if $A \notin m$ then $i^{*}{ }_{m}(A)=\Phi$ and $c^{*}{ }_{m}(X-A)=X$. So, $i^{*}{ }_{m}(A) \subset i_{m}(A), c_{m}(A) \subset c^{*}{ }_{m}(A)$. Let $X=\{a, b, c\}$ and $m=\{\Phi, X,\{a\},\{b, c\}\}$ then $i^{*}{ }_{m}(\{a\})=\{a\} \supset \Phi=$ $i^{*}{ }_{m}(\{a, b\})$ and $c^{*}{ }_{m}(\{b, c\})=\{b, c\} \subset X=c^{*}{ }_{m}(\{b\})$, hence $i^{*}$ and $c^{*}$ are not monotonic on the power set $P(X)$ of the underlying set $X$. Now let $x \notin$ $X-i^{*}{ }_{m}(X-A) \Leftrightarrow x \in i^{*}{ }_{m}(X-A) \Leftrightarrow x \in X-A \in m \Leftrightarrow X-A \in M(x) \Leftrightarrow x \notin$ $c^{*}{ }_{m}(A)$. Thus $c^{*}{ }_{m}(A)=X-i^{*}{ }_{m}(X-A)$ and similarly $i^{*}{ }_{m}(A)=X-c^{*}{ }_{m}(X-A)$ for all $A \subset X$. The purpose of the definitions of $i^{*}{ }_{m}$ and $c^{*}{ }_{m}$ is the fact that $i^{*}{ }_{m}(A)=A \Rightarrow A \in m$ and $c^{*}{ }_{m}(A)=A \Rightarrow X-A \in m$. Thus we have

Theorem 3.4. Let $m$ be a minimal structure on $X$. Then

1. $i^{*}{ }_{m}(A)=i_{m}(A) \forall A \in m$

2. $c^{*}{ }_{m}(X-A)=c_{m}(X-A) \forall A \in m$

3. $i^{*}{ }_{m}(A)=\Phi$ and $c^{*}{ }_{m}(X-A)=X$ whenever $A \in P(X)-m$.

4. $i^{*}{ }_{m}(A) \subset i_{m}(A)$, and $c_{m}(A) \subset c^{*}{ }_{m}(A) \forall A \subset X$.

5. $i^{*}{ }_{m}$ and $c^{*}{ }_{m}$ are monotonic on $m$ but not on the power set $P(X)$ (if $m \neq$ $P(X))$ of the underlying set $X$.

6. $c^{*}{ }_{m}(A)=X-i^{*}{ }_{m}(X-A) \forall A \subset X$.

7. $i^{*}{ }_{m}(A)=A \Rightarrow A \in m$ and $c^{*}{ }_{m}(A)=A \Rightarrow X-A \in m$.

Theorem 3.5. An MSS $(X, m)$ is $m$-regular if for any $x \in U \in m$ there exists $V \in m$ so that $x \in V \subset c^{*}{ }_{m}(V) \subset U$.

Proof. Now in an MSS $(X, m)$, let for any $x \in U \in m$ there exists $V \in m$ so that $x \in V \subset c^{*}{ }_{m}(V) \subset U$. Let $x$ be any point of $X$ and $C$ be any $m$-closed set not containing $x$, so $x \in X-C$ and $X-C$ is an $m$-open set and therefore by the hypothesis there exists $m$-open set $V$ such that $x \in V \subset c^{*}{ }_{m}(V) \subset X-C \Rightarrow$ $x \in V$ and $C \subset X-c^{*}{ }_{m}(V)=T$ (say). Then since $V \cap T=\phi$ hence the MSS $(X, m)$ is $m$-regular. Thus an $\operatorname{MSS}(X, m)$ is $m$-regular if for any $x \in U \in m$ there exists $V \in m$ so that $x \in V \subset c^{*}{ }_{m}(V) \subset U$

Example 3.2. The MSS $(\mathbb{R}, m)$ where $m$ is the collection of all right and left open rays including $\phi$ and $\mathbb{R}$ shows that the condition in Theorem 3.5 is not necessary.

Theorem 3.6. If an $M S S(X, m)$ is $m$-regular then for any $x \in U \in m$ there exists $V \in m$ so that $x \in V \subset c_{m}(V) \subset U$. 
The $\operatorname{MSS}\left(X, m_{1}\right)$ in the Example 3.1 ensures that this condition is not sufficient. We search a necessary as well as sufficient condition for an MSS $(X, m)$ to be $m$-regular.

Theorem 3.7. An MSS $(X, m)$ is $m$-regular if and only if for any point $x \in X$ and any $m$-open set $U$ containing $x$ there exist $m$-open set $V$ and $m$-closed set $V^{*}$ so that $x \in V \subset V^{*} \subset U$.

Proof. Let an MSS $(X, m)$ be $m$-regular, $x$ be any point in $X$ and $U$ be any $m$-open set containing $x$. Then $X-U$ is $m$-closed set not containing $x$. So, there are $m$-open sets $E$ and $F$ such that $x \in E,(X-U) \subset F$ and $E \cap F=\phi$. Hence $E \subset(X-F) \subset U$. Taking $V=E$ and $V^{*}=(X-F)$ we have $V$ is $m$-open, $V^{*}$ is $m$-closed and $x \in V \subset V^{*} \subset U$. Conversely, let for any point $x \in X$ and any $m$-open set $U$ containing $x$ there exists $m$-open set $V$ and $m$-closed set $V^{*}$ so that $x \in V \subset V^{*} \subset U$. Let $x \in X$ and $E$ be any $m$-closed set not containing $x$. Then $x \in(X-E)$ and $(X-E)$ is $m$-open. Hence by the hypothesis there is an $m$-open set $V$ and an $m$-closed set $V^{*}$ so that $x \in V \subset V^{*} \subset(X-E)$. Therefore $E \subset\left(X-V^{*}\right)$, also $V \cap\left(X-V^{*}\right) \subset V \cap(X-V)=\phi$ hence $V \cap\left(X-V^{*}\right)=\phi$ which implies $(X, m)$ is $m$-regular.

Definition 3.6. An MSS $(X, m)$ is called $m$-normal if for any two disjoint $m$-closed sets $C$ and $D$ there exists two disjoint $m$-open sets $U$ and $V$ so that $C \subset U$ and $D \subset V$.

Theorem 3.8. Let $C$ be any $m$-closed set and $U$ be any $m$-open set containing $C$. Then there exists an $m$-open set $V$ so that $C \subset V \subset c^{*}{ }_{m}(V) \subset$ $U \Rightarrow \operatorname{MSS}(X, m)$ is $m$-normal $\Rightarrow$ there exists an $m$-open set $V$ so that $C \subset$ $V \subset c_{m}(V) \subset U$.

Theorem 3.9. A necessary and sufficient condition for an MSS $(X, m)$ is $m$-normal if for any $m$-closed set $C$ and any $m$-open set $U$ containing $C$ there is an $m$-open set $V$ and an $m$-closed set $V^{*}$ such that $C \subset V \subset V^{*} \subset U$.

Definition 3.7. A function $f: X \rightarrow Y$ from an $\operatorname{MSS}\left(X, m_{X}\right)$ into an MSS $\left(Y, m_{Y}\right)$ is said to be $M$-continuous at a point $x \in X$ if for any $V \in M(f(x))$, there is $U \in M(x)$ such that $f(U) \subset V ; f$ is called $M$-continuous if it is so at each point of its domain.

Theorem 3.10. For any function $f: X \rightarrow Y$ from an $\operatorname{MSS}\left(X, m_{X}\right)$ into an $\operatorname{MSS}\left(Y, m_{Y}\right)$, as in general case, it is observed that the following are equivalent:

(1) $f$ is $M$-continuous.

(2) $f\left(c_{m_{X}}(A)\right) \subset c_{m_{Y}}(f(A))$ for $A \subset X$. 
(3) $c_{m_{X}}\left(f^{-1}(B)\right) \subset f^{-1}\left(c_{m_{Y}}(B)\right)$ for $B \subset Y$.

(4) $f^{-1}\left(i_{m_{Y}}(B)\right) \subset i_{m_{X}}\left(f^{-1}(B)\right)$ for $B \subset Y$.

Definition 3.8. A function $f: X \rightarrow Y$ from an $\operatorname{MSS}\left(X, m_{X}\right)$ into an $\operatorname{MSS}\left(Y, m_{Y}\right)$ is said to be $M^{*}$-continuous if for every $B \in m_{Y}, f^{-1}(B) \in m_{X}$.

Obviously $M^{*}$-continuity implies $M$-continuity but the converse is not true as seen in the following Example 3.3.

Example 3.3. Let $X=Y=\{a, b, c\}, m_{X}=\{\phi, X,\{a\},\{b\}\}$, and $m_{Y}=$ $\{\phi, X,\{a, b\}\}$ then the identity function is $M$-continuous but not $M^{*}$-continuous.

If $x \in i_{m}^{*}(A)$ then there is a $U \in M(x)$ contained in $A$ but $x \in c^{*}{ }_{m}(A)$ does not mean that every $V \in M(x)$ intersects A as shown in the following Example 3.4 .

Example 3.4. Let $X=\{a, b, c\}, m=\{\phi, X,\{a\},\{a, b\},\{b, c\}\}$ and $A=$ $\{b\}$ then, $a \in c^{*}{ }_{m}(A)=X$ and $V=\{a\} \in M(a)$ but $V \cap A=\phi$.

This fact together with the non-monotonicity of the operators $i^{*}$ and $c^{*}$ cause the difference between $M$-continuity and $M^{*}$-continuity.

Theorem 3.11. Let $f: X \rightarrow Y$ be a function from an $M S S\left(X, m_{X}\right)$ into an $\operatorname{MSS}\left(Y, m_{Y}\right)$, then the following are equivalent:

(i) $f$ is $M^{*}$-continuous.

(ii) $f^{-1}\left(i_{m_{Y}}^{*}(B)\right) \subset i_{m_{X}}^{*}\left(f^{-1}(B)\right)$ for $B \subset Y$.

(iii) $c_{m_{X}}^{*}\left(f^{-1}(B)\right) \subset f^{-1}\left(c_{m_{Y}}^{*}(B)\right)$ for $B \subset Y$.

If further $f$ is a bijection, the necessity of it is given below, then all these three statements are equivalent to

(iv) $f\left(c_{m_{X}}^{*}(A)\right) \subset c_{m_{Y}}^{*}(f(A))$ for $A \subset X$.

Example 3.5. Let $X=\{a, b, c\}, Y=\{1,2\}, m_{X}=\{\phi, X,\{b\}\}, m_{Y}=$ $\{\phi, Y,\{2\}\}$ and $f: X \rightarrow Y$ be a function defined by $f(a)=f(c)=1, f(b)=2$; $f$ is $M^{*}$-continuous hence (iii) holds but $f\left(c_{m_{X}}^{*}(A)\right)=f(X)=\{1,2\} \nsubseteq\{1\}=$ $c_{m_{Y}}^{*}(\{1\})=c_{m_{Y}}^{*}(f(A))$ where $A=\{c\}$ shows that (iv) does not hold. Also let $X=\{a, b, c\}, Y=\{1,2,3\}, m_{X}=\{\phi, X,\{b\}\}$ and $m_{Y}=\{\phi, Y,\{2\}\}$. Then the function $f: X \rightarrow Y$ defined by $f(a)=f(b)=1, f(c)=2$ is not $M^{*}$-continuous, so (iii) can not be true but it can be verified that (iv) holds.

Definition 3.9. Intersection of any right open ray $(a, \infty)$ in the linearly ordered set of real numbers with $[0,1]$ is called a right open ray in $[0,1]$; similarly define left open ray in $[0,1]$. A function $f: X \rightarrow[0,1]$ from an $\operatorname{MSS}\left(X, m_{X}\right)$ into the subspace $[0,1]$ of $\mathbb{R}$ with usual topology is said to be $m_{X}$-upper-semicontinuous (resp. $m_{X}$-lower-semi-continuous) [1] at a point $x \in X$ if for any 
left (resp. right) open ray $R$ in $[0,1]$ containing $f(x)$, there is $U \in M(x)$ such that $f(U) \subset R ; f$ is called $m_{X}$-upper-semi-continuous (resp. $m_{X}$-lower-semicontinuous) if it is so at each point of its domain.

Example 3.6. Let $m_{l}$ and $m_{r}$ be the collections of all left-open rays and right-open rays respectively in $[0,1]$ together with $\phi$ and $[0,1]$. Then the identity mapping $e$ on $[0,1]$ is $m_{l}$-upper-semi-continuous, $m_{r}$-lower-semi-continuous and $m_{l} \cup m_{r}$-upper-lower-semi-continuous but neither it is $m_{l}$-lower-semi-continuous nor $m_{r}$-upper-semi-continuous even $e:\left([0,1], m_{l} \cup m_{r}\right) \rightarrow[0,1]$ is not $M$ continuous.

We end with the following generalization of Urysohn's lemma.

Theorem 3.12. For any pair of disjoint $m$-closed sets $C$ and $D$ in an $m$-normal MSS $(X, m)$ there is a $m$-upper-lower-semi-continuous function $f$ : $X \rightarrow[0,1]$ so that $f(x)=0 \forall x \in C$ and $f(x)=1 \forall x \in D$.

Proof. Let $C$ and $D$ be any disjoint $m$-closed sets in an $m$-normal MSS $(X, m), V_{1}=X-D$ and $V_{0}{ }^{*}=C$. Since the $m$-closed set $V_{0}{ }^{*}$ is contained in the $m$-open set $V_{1}$, therefore, by using $m$-normality there is an $m$-open set $V_{\frac{1}{2}}$ and an $m$-closed set $V_{\frac{1}{2}}{ }^{*}$ so that $V_{0}{ }^{*} \subset V_{\frac{1}{2}} \subset V_{\frac{1}{2}}{ }^{*} \subset V_{1}$. Applying the hypothesis on $(X, m)$ to each pair $V_{0}{ }^{*}, V_{\frac{1}{2}}$ and $V_{\frac{1}{2}}{ }^{*}, V_{1}$, we have $m$-open sets $V_{\frac{1}{4}}, V_{\frac{3}{4}}$ and $m$-closed sets $V_{\frac{1}{4}}{ }^{*}, V_{\frac{3}{4}}{ }^{*}$ so that $V_{0}{ }^{*} \subset V_{\frac{1}{4}} \subset V_{\frac{1}{4}}{ }^{*} \subset V_{\frac{1}{2}} \subset V_{\frac{1}{2}}{ }^{*} \subset V_{\frac{3}{4}} \subset V_{\frac{3}{4}}{ }^{*} \subset V_{1}$. Continuing this process one can define $m$-open sets $V_{s}, V_{t}$ and $m$-closed sets $V_{s}{ }^{*}$, $V_{t}^{*}$ for any dyadic rational $s$ and $t$ in $[0,1]$ of the form $\frac{k}{2^{n}}, k=1,2,3 \ldots \ldots .2^{n}-1$ and $n \in \mathbb{N}$ so that $s<t \Rightarrow V_{0}{ }^{*} \subset V_{s} \subset V_{s}{ }^{*} \subset V_{t} \subset V_{t}{ }^{*} \subset V_{1}$. If $s$ is any other dyadic rational, let $V_{s}=\phi$ for $s \leq 0, V_{s}=X$ for $s>1$, and $V_{s}{ }^{*}=\phi$ for $s<0, V_{s}^{*}=X$ for $s \geq 1$. Now consider a function $f: X \rightarrow[0,1]$ defined by $f(x)=\inf \left\{s ; x \in V_{s}\right\}=\inf \left\{s ; x \in V_{s}^{*}\right\} \forall x \in X$. Then $f(x)=0, \forall x \in C$ and $f(x)=1, \forall x \in D$. Definition of the function $f$ and construction of the sets $V_{s}$ and $V_{s}^{*}$ for dyadic rational number $s$ show that $x \in V_{s}{ }^{*} \Rightarrow f(x) \leq s$ and $x \notin V_{s} \Rightarrow f(x) \geq s$. Now for the ray [0,1] (which is left as well as right open ray in $[0,1]) U$ is an $m$-open set containing $x_{0}$ so that $f(U) \subset[0,1]$ where $U=V_{1}$ if $f\left(x_{0}\right)=0$ and if $f\left(x_{0}\right)=1$ then $U=X-V_{0}^{*}$. For any left open ray $[0, d)$ in $[0,1]$ containing $f\left(x_{0}\right)$ choose a dyadic rational $q$ such that $f\left(x_{0}\right)<q<d$ and consider the $V_{q}$. Since $0<q<1$ therefore $V_{q}$ is $m$-open set containing $x_{0}$ because otherwise $f\left(x_{0}\right) \geq q$. Also $f\left(V_{q}\right) \subset[0, d)$ since $x \in V_{q} \Rightarrow f(x) \leq q$. And for any right open ray $(c, 1]$ in $[0,1]$ containing $f\left(x_{0}\right)$ select a dyadic rational $p$ such that $c<p<f\left(x_{0}\right)$ then, $X-V_{p}{ }^{*}$ is an $m$-open set containing $x_{0}$ so that $f\left(X-V_{p}^{*}\right) \subset(c, 1]$. 
Remark. Here we have a comment on Theorem 3.12. In the Theorem 3.12 , if the minimal structure $m$ is a topology then $C$ and $D$ become disjoint closed sets in the corresponding normal topological space, $m$-upper-lower-semicontinuity corresponds to continuity and Urysohn's lemma of point set topology will follow. Thus it can be stated that the Theorem 3.12 is not a translation of Urysohn's lemma, it is a generalization of Urysohn's lemma.

\section{References}

[1] N. Levine, Semi-open sets and semicontinuity in topological spaces, Ams. Math. Monthly, 70 (1963), 36-41.

[2] V. Popa, T. Noiri, On M-continuous functions, Anal. Univ. "Dunarea de Jos" Galati, Ser. Mat. Fiz. Mec. Teor., Fasc. II, 18, No. 23 (2000), 31-41.

[3] Carlos Carpintero, Ennis Rosas, Margot Salas, Minimal structures and separations properties, Int. J. Pure Appl. Math., 34, No. 4 (2007), 473488.

[4] A. Pushpalatha and E. Subha, Strongly generalized closed sets in minimal structures, Int. Journal of Math. Analysis, 3, No. 26 (2009), 1259-1263.

[5] R. Parimelazhagan, K. Balachandran, N. Nagaveni, Weakly generalized closed sets in minimal structure, Int. J. Contemp. Math. Sciences, 4, No. 27 (2009), 1335-1343.

[6] Sunisa Buadong, Chokchai Viriyapong, Chawalit Boonpok, On generalized topology and minimal structure spaces, Int. Journal of Math. Analysis, 5, No. 31 (2011), 1507-1516.

[7] W. K. Min, Y. K. Kim, M*-Continuity and product minimal structure on minimal structures, Int. J. Pure Appl. Math., 69, No. 3 (2011), 329-339. 
